\title{
25 Research Soure \\ Examining Notions of Uncertainty in the Healthcare Setting: A Qualitative Study
}

Veronica Meawad ( $\nabla$ veronica.meawad@yale.edu)

Yale University School of Medicine https://orcid.org/0000-0002-1552-5892

Liana Fraenkel

Yale University School of Medicine

Research article

Keywords: Uncertainty, probability, risk communication, uncertainty perception, epistemic, aleatory, visual aids

Posted Date: December 15th, 2019

DOI: https://doi.org/10.21203/rs.2.18895/v1

License: (1) This work is licensed under a Creative Commons Attribution 4.0 International License. Read Full License 


\section{Abstract}

Background: Shared decision-making relies on the premise that individuals are well-informed regarding the benefits and harms of all available treatment options before coming to a decision regarding their healthcare. There is often significant aleatory and epistemic uncertainty associated with proposed risks and benefits, but prior studies have demonstrated that uncertainty is rarely communicated in providerpatient interactions. Additionally, there has been conflicting evidence regarding the use of visual aids as a method to improve understanding of uncertainty.

Methods: We conducted a qualitative, think-aloud study to examine how individuals understand uncertainty as it relates to the probability of medication risks and benefits. We used a within-subjects design and presented individuals with two hypothetical medications after which they received either no additional information, an icon array, or a spinner. We performed individual in-depth interviews following a semi-structured interview guide. Transcripts were analyzed using the constant comparative method of grounded theory.

Results: We reached thematic saturation after 20 patient interviews. The participants' mean (SD) age was 64.6 (18); $60 \%$ male, $84 \%$ were non-Hispanic Whites, $10 \%$ Blacks, and $60 \%$ had a college education or higher. Several themes regarding difficulties understanding uncertainty emerged: 1) Rejection of uncertainty; 2) Failure to distinguish between epistemic and aleatory uncertainty; 3) Difficulty distinguishing between different magnitudes of probabilities; 5) Reduction of uncertainty by manipulation of the scenario to eliminate choices. The use of visual aids did not appear to facilitate understanding of uncertainty in most cases.

Conclusions: Providers must understand whether and how patients interpret probabilistic information in order to best communicate with them in the healthcare setting. Features such as "talk-back" should be used to ensure reasonable understanding of uncertainty during shared decision-making encounters.

\section{Background}

Shared decision-making relies on the premise that individuals are well-informed regarding the benefits and harms of all available treatment options before coming to a decision regarding their healthcare. Therefore, it only makes sense that discussing the uncertainties associated with different options is a critical part of informed decision-making. The evidence, however, suggests otherwise. ${ }^{1,2}$ An analysis of over one-thousand clinical encounters by primary care physicians and surgeons found that only 16 to $18 \%$ of discussions met the minimum criteria for an informed decision. ${ }^{2}$ Discussion of the uncertainty of risks and benefits was only done $1 \%$ of the time for basic decisions and $16 \%$ of the time for complex decisions. $^{2}$

Several different approaches have been put forth to classify uncertainty. Paul Han, in his conceptual taxonomy, outlined three main sources of uncertainty in healthcare: probability, ambiguity, and 
complexity. ${ }^{3}$ Many have argued that these sources should be clearly stated and differentiated when communicating for purposes of medical decision-making. ${ }^{1,3}$ Uncertainty can also be conceptualized as aleatory, or first-order uncertainty, which is inherent and related to the probability of future outcomes; and epistemic, or second-order uncertainty, which is due to limits in data or knowledge. ${ }^{4-6}$ Aleatory uncertainty is influenced by stochastic behaviors, whereas epistemic uncertainty is better known as ambiguity due to lack of credibility, reliability, or inadequacy of probability information.

It is well established that people have a preference against unknown or ambiguous versus known probabilities. ${ }^{7,8}$ In the field of decision psychology, the term ambiguity aversion has been used to describe the phenomenon in which people tend to view risks negatively when confronted by ambiguous information about risks, which then leads to decision-making avoidance. ${ }^{9}$ For example, intervention studies have shown that informing individuals about the uncertainties surrounding cancer screening measures decreases their interest in screening. ${ }^{10,11}$ Another study examining the association between uncertainty and judgement extremity, i.e., the degree to which probabilistic beliefs approach 0 or 1 , demonstrated a tendency for judgements to be more extreme when they were viewed as epistemic and less extreme when they were seen as more aleatory. ${ }^{12}$ Others have argued that ambiguity aversion can actually be adaptive as it ensures that people do not have a false sense of security. ${ }^{6}$ From these studies, it is evident that individuals' thoughts regarding uncertainty and how they perceive uncertainty influences their judgement and future decisions.

There are various ways of communicating uncertainty. Some use descriptive phrases, such as "highly certain" versus "fairly certain" to communicate uncertainty. ${ }^{1,3}$ This approach has the advantage of using familiar terms, but the interpretation of verbal phrases varies significantly. ${ }^{1,13-15}$ Communicating uncertainty using numbers, while more precise, is also problematic because many individuals have difficulty interpreting numeric information. ${ }^{1,16-19}$ Those with low numeracy, or the ability to understand and apply numeric information, are more susceptible to framing effects, denominator neglect, and ratio bias. ${ }^{18,19}$ Their decisions are also more likely to be influenced by nonnumeric factors, such as emotions, mood states, and trust or distrust of physicians. ${ }^{20}$

The use of visual aids, such as icon arrays, graphs, and explanatory images, ${ }^{21}$ has been endorsed as a method to improve understanding of uncertainty, especially for those with lower levels of numeracy. Studies, however, have been conflicting. While some have shown that graphics improve understanding among those with low numeracy, ${ }^{22-24}$ others have not found any benefit, and some have found that visual aids may actually decrease understanding among those with low numeracy and low graphic literacy. ${ }^{24-28} \mathrm{~A}$ recent paper by Eyler et al. proposed using a continuous "spinner" to communicate risk based on studies in children demonstrating improved understanding of probabilities when shown continuous versus discrete patterns. ${ }^{29}$ The study found that both the spinner and icon array increased subjects' risk knowledge, although the effect was not modified by numeracy. ${ }^{29}$ 
Our aim was to examine how individuals understand uncertainty as it relates to the probability of medication risks and benefits, and to specifically analyze their understanding of epistemic and aleatory uncertainty. Additionally, given the conflicting evidence regarding the effectiveness of visual aids in increasing understanding of uncertainty, we aimed to study how people think about both the icon array and spinner.

\section{Methods}

We conducted a qualitative, think-aloud study using a semi-structured interview guide. Participants were recruited from the inpatient wards of a tertiary care hospital. Inclusion criteria included English speaking adult inpatients who were not mentally altered through the use of sedating medications or by their illness and able to answer questions out loud. Charge nurses on the medical floors directed us to appropriate individuals. Verbal consent was obtained prior to participation. The participants were presented with two hypothetical medications. The first scenario involved a medication with a $20 \%$ risk of adverse effect and the second involved a medication with a $20 \%$ chance of benefit. Using block randomization, generated by Sealed Envelope (https://www.sealedenvelope.com/), with six subjects per block, participants were randomized to receiving either the risk scenario or benefit scenario first. All participants received both scenarios. Also using block randomization with six subjects per block, generated by the same means, they were randomized to receiving either no additional information, an icon array, or a spinner with risk or benefit probability information associated with each scenario.

We conducted individual in-depth interviews following a semi-structured interview guide. Participants were instructed to think and reason out loud, saying everything that came to mind. The initial prompt read as follows: "We are interested in what you think about as you work through some questions that I will give you. In order to do this, I'm going to ask you to think and reason out loud, saying everything that passes through your mind while you read the question and as you reason through the answer. Just act as if you are alone in the room speaking to yourself. It is most important that you keep talking. If you are silent for a long period of time, I may ask you to talk."

Scenarios (presented in random order) were read out loud by facilitators but a hard copy was provided in order for participants to follow along.

Risk Scenario:

"Imagine that you have been suffering from arthritis pain in your knees for several years. Your doctor tells you about a medication which can be used to decrease your pain. It is taken as a pill once a day. The medication is covered by your insurance and does not interact with your other medications. The medication works in the majority of people, but causes stomach problems, such as stomach upset, nausea, or diarrhea, in $20 \%$ of people who take it."

Benefit Scenario: 
"Imagine that you have been suffering from arthritis pain in your knees for several years. Your doctor tells you about a medication which can be used to decrease your pain. It is taken as a pill once a day. The medication is covered by your insurance and does not interact with your other medications. The medication is well-tolerated in the majority of people and decreases pain in $20 \%$ of people who take it."

After the scenarios were read, participants were asked questions such as "What are the thoughts that run through your mind as you hear about this medication?" and "What does $20 \%$ of people mean to you?" If participants were quiet for a long period of time, they were instructed to keep talking. Other prompts included, "What do you think of this medication?" and "What do you think about the chance of getting stomach problems." All interviews were audiotaped and subsequently transcribed verbatim.

Transcripts were analyzed separately by both authors using the constant comparative method of grounded theory, first outlined by Glaser \& Strauss (1967), with techniques further explained by others (Strauss \& Corbin, 2008; Kolb, 2012). Data analysis included three phases: open coding, axial coding, and selective coding (Strauss \& Corbin, 2008). The open coding step aimed to identify different categories and properties within the collected data. This involved reviewing all transcripts and placing the data into fine categories. This phase of analysis was done concurrently with additional data collection. In the next phase, axial coding, the numerous, different categories were weaved together to form larger categories. In order to do this, both authors came together with their separately-found categories from the open coding stage and pieced together broader categories. In the final stage, selective coding, the categories generated by axial coding were refined and core themes were selected (Strauss \& Corbin, 2008; Kolb, 2012). Coding ended with thematic saturation, or when both authors were unable to identify additional core themes within the data.

\section{Results}

We reached thematic saturation after interviewing 20 patients. The participants' mean (SD) age was 64.6 (18); $60 \%$ male, $84 \%$ were non-Hispanic Whites, $10 \%$ Blacks, and $60 \%$ had a college education or higher. Participants were recruited from both medical and surgical medical units. Several themes regarding difficulties understanding uncertainty emerged.

\section{Theme 1:Rejection of uncertainty}

Some participants rejected uncertainty altogether, and instead focused on the possible mechanisms of action underlying an outcome once it had already occurred. One participant said,

"Well I kinda think there's a reason for it. I don't know if I would call it random or chance. There's a reason why it's not working." (Interview \#2)

Others stated,

"I mean if you feel like you have the tendency, that's not random, you know." (Interview \#5) 
"It's ridiculous because it's not a chance. You either get it or you don't. You have a predisposition for it and you don't know it and then you find out when you take it." (Interview \#19)

These participants focused on the reasons why someone would or would not experience a certain risk or a benefit, whether it was an unknown predisposition, an interaction with another medication, allergic reaction, etc. They used terms such as predisposition, tendency, and reason. They did not acknowledge the role that "chance" had in the development of a risk or benefit.

For these participants, neither the icon array nor spinner appeared to facilitate acceptance of uncertainty: "According to the diagram [icon array], it looks good, but you don't know until you know." (Interview \#15) "I don't like it [the spinner]. Yeah. It's just a- it's like a toy... It doesn't have a brain. This is cardboard, I mean... It can't predict. It's not god." (Interview \#6)

"Well there are some people who would get stomach problems. So there's definitely- there definitely are people. But they're not talking about those people. They're talking about me." (Interview \#2)

\section{Theme 2:Failure to distinguish between aleatory and epistemic uncertainty}

Several participants interpreted uncertainty as mainly epistemic. These individuals felt that expert knowledge could reduce or eliminate uncertainty and/or that one's own knowledge from prior experiences could do the same. Illustrative examples include:

"I think with enough information, you could answer in advance whether it would relieve your pain." (Interview \#2)

"I think as much as experts know, they don't know everything. But you have to trust that they're gonna know that." (Interview \#2)

"They know something that I don't know." (Interview \#17)

"Uh, we're not, um, scientists that we can predict what a pill is gonna do." (Interview \#6)

These individuals and several others strongly felt that experts possessed knowledge that would help them predict whether they would experience a certain risk or benefit. They repeated words such as scientists, predict, and knowledge.

Additionally, many others felt that personal knowledge from prior experiences could reduce uncertainty. For example:

"I would say I would probably not get stomach problems at all, due to the fact that in the past, any medication I have taken, I really have never had a problem with." (Interview \#17) 
"The other question is that you know your body and you know how it responds to new things, so you do have that information or knowledge from past experiences." (Interview \#20)

These participants correctly believed that the knowledge they possessed from their own past experiences could decrease uncertainty. If they had had a similar side effect in the past, then they seemed to believe they had a higher chance of experiencing it again and vice versa. However, they tended to overweight this information and to classify their risk as either present or not present.

\section{Theme 3: Difficulty distinguishing between different magnitudes of probabilities}

Some participants accurately distinguished between different magnitudes of probabilities while others did not. Of those who were able to differentiate between magnitudes, some did not find the visual aids helpful.

"I like math and I'm good at math and you told me 20 percent. For me, it's not very helpful [the spinner]. Not that it's not helpful, it's just maybe if it was somebody... Like somebody, you know, I guess maybe somebody who's more of a visual person?" (Interview \#9)

"I don't think it [the spinner] does a whole lot unless you really don't know percentages or things like that. But they use it for breaking down costs and budgets and all kinds of other things. If I don't know how much 20 percent is, then I guess I could look at this and have a clue, but so maybe for some people it'll help but, I don't find it a big help." (Interview \#16)

Furthermore, among those who did differentiate between magnitudes, some translated the numeric information into ordinal categories:

"You know, I got a good chance of not getting sick. I got a smaller chance of possibly getting sick." (Interview \#7)

"I mean, you know, there's 80 percent over here and then this little 20 percent." (Interview \#10)

"I would say little [the risk associated]." (Interview \#6)

These individuals constructively dealt with probabilistic information by creating mental representations of categories of risk. Others, however, categorized any risk as $50 \%$ :

"You got a fifty-fifty chance basically that it's going to work or it's not, even though it's only 20 percent demographic but, you know, there's that fifty-fifty chance it's going to take." (Interview \#1)

"I say 50\% [chance of medication working]." (Interview \#13)

They ignored the numeric probability that was given and deemed any risk as having a $50 \%$ chance of occurring. 
For one participant, seeing the icon array appeared to hamper, rather than facilitate, understanding of possible outcomes:

"No I guess it would-it could. It's not totally out of the question. But if you have to base it on, you know, statistics here, um, oh boy. Now I get myself all messed up." (Interview \#2)

\section{Theme 4: Reduction of uncertainty by manipulation of the scenario to eliminate choices}

Another major theme that emerged was one in which participants reduced uncertainty by manipulating the scenario to eliminate the need to attend to probabilities at all. In these cases, participants reduced the options available to a single rational choice by revising the severity of symptoms. For example,

"I would say if it's sort of a life or death situation, then you don't care as much about the risk." (Interview \#3)

"Well I think if people are really suffering that-and if that's the only thing available to them then they should do it." (Interview \#12)

"However, being somebody who really suffers from this, I would take the risk and take it anyway because it's worth it to try." (Interview \#2)

By increasing pain or suffering, these participants eliminated the option of not accepting treatment. Words and phrases such as suffering and life or death were common in these individuals.

\section{Discussion}

Understanding uncertainty is critical to being able to participate in medical decision making. This study uncovered several themes regarding how patients view and understand uncertainty in the healthcare setting. In general, we found that many individuals have substantial difficulty understanding uncertainty as it relates to medication risks and benefits, and in many cases, that visual aids did not aid in understanding. First, we discovered that some individuals reject uncertainty altogether and do not understand the concept of chance as it applies to future events. These people seem to confuse the concepts of knowing why something happened in the past with knowing if something will happen in the future. In other words, they conflate the ideas of understanding the underlying mechanism with being able to predict a future occurrence. This is of course an inaccurate way of thinking, since every future outcome has an inherent chance regardless of whether or not the reason is understood.

Second, among those who did not reject uncertainty, we found that several participants interpreted the uncertainty as mainly epistemic, rejecting the inherent probability, or aleatory uncertainty, associated with developing a certain risk or benefit. Of these, some individuals reduced uncertainty by drawing upon knowledge from their own prior experiences. In many cases, it is true that if one experienced a certain side effect in the past, they have a higher chance of experiencing it again, and therefore this information can be used to narrow one's confidence interval. However, participants do this based on their own illness 
perceptions, which may or may not be accurate, and tend to generalize their experience with one medication to others. If inaccurate, this type of reasoning may lead to the formation of erroneous associations between past events and the likelihood of future outcomes.

Similarly, several individuals believed that expert knowledge could reduce uncertainty. They believed that the uncertainty of specific medical outcomes could be reduced or eliminated by gathering more information, particularly from experts. This notion has bearings on provider-patient interactions and medical decision making. If patients perceive uncertainty as mainly epistemic, a search for additional information to eliminate uncertainty may lead to dissatisfaction with providers, doctor-shopping, and unnecessary testing. These results highlight the need for future research to determine how to best communicate differences between aleatory and epistemic uncertainty.

Of the participants who did appreciate uncertainty, some ignored the numeric or visual information that was provided to them. They understood that the icon array and spinner were describing past events, but that future outcomes were still unknown and could not be extrapolated from the figures. This could be a possible explanation as to why those with low numeracy still do not have a better understanding of uncertainty after the use of visual aids. We also saw that those with seemingly high numeracy, did not feel the need to use the visual aids. These results are consistent with previous studies that have found that those with high numeracy interpret numeric information without the need for visual aids, ${ }^{22,30}$ and those with low numeracy have difficulty interpreting graphical information. ${ }^{20,25,26}$ In total, our study highlights the need for future work to determine whether patients with moderate levels of numeracy and/or graphic literacy maybe those most likely to benefit from visual aids.

Consistent with fuzzy-trace theory, some vocalized accurate ordinal representations of risk: "You know, I got a good chance of not getting sick. I got a smaller chance of possibly getting sick," highlighting how gist-based intuitive ordinal reasoning often suffices to produce rational solutions. ${ }^{31}$ Several participants used gist-based reasoning to place themselves into different categories of risk. In some cases, reasoning included a component of uncertainty (varying degrees of risk), whereas in others it resulted in participants concluding that they would either develop or not develop an outcome.

As noted in other studies, we observed a tendency to interpret any probability as "fifty-fifty." The use of the verbal phrase "fifty-fifty" reflects lack of knowledge and a way of expressing epistemic uncertainty, as opposed to the actual quantity $50 \%{ }^{32,33}$ It is also correlated with lower numeracy, younger age, and lower levels of education. ${ }^{34}$ Others have shown that those who say " $50 \%$ " are more likely to also say "I don't know" than those who use other numbers. ${ }^{35}$ Notably, in our study, participants described chance as fiftyfifty even after having been told through verbal and visual aids that the probabilities were magnitudes other than $50 \%$, further highlighting the limitations of visual aids in those with low numeracy and/or graphic literacy.

Finally, we also found that some individuals reduced uncertainty by eliminating choice altogether. This type of rationalizing is reasonable for individuals in extreme circumstances, such as those with life- 
threatening medical conditions who need lifesaving interventions. It is adaptive for those individuals to eliminate choice in order to make a decision which is life-preserving. Moreover, in critical situations, there is often a single dominant strategy and therefore no actual decision to be made. In our study, though, in which the ailment was less serious (i.e. knee pain), some participants modified the scenario to one in which clinical need was greater than we had originally described in order to eliminate the need to make a difficult choice.

There are several limitations to our study. Generalizability may be limited, since our population was mostly comprised of older white inpatients at one tertiary care hospital. It would be interesting to conduct future think aloud studies in different settings and with minority populations to assess if the same major themes hold. We also would have benefitted from examining patients' understandings across different hypothetical scenarios.

\section{Conclusion}

Our study highlights the need to understand whether and how our patients interpret probabilistic information in order to best communicate with them in the healthcare setting. We found that individuals interpret uncertainty in different ways. Some reject it altogether, while others aim to reduce it using various means. Based on our study, there is reason to believe that visual aids are less useful for those at the extremes of high and low numeracy. Providers should use features such as "talk back" to ensure reasonable understanding of uncertainty during shared decision-making encounters.

\section{Declarations}

Ethics approval and consent to participate: Ethics approval for this study was obtained by Yale University Institutional Review Board. All participants were consented prior to their participation and given the option to withdraw at any time.

Consent for publication: Not applicable

Availability of data and materials: The data used and analyzed during the current study are available from the corresponding author on reasonable request.

Competing interests: The authors declare that they have no competing interests.

Funding:Research reported in this publication was also supported by the National Institute of Arthritis and Musculoskeletal and Skin Diseases, part of the National Institutes of Health, under Award Number AR060231-06 (Fraenkel). The content is solely the responsibility of the authors and does not necessarily represent the official views of the National Institutes of Health. The authors do not have any conflicts of interest related to the content of this manuscript. 
Authors' contributions: VA conducted the patient interviews. Both VA and LF analyzed and coded the transcripts. Both VA and LF were major contributors in writing the paper. Both authors read and approved the final manuscript.

Acknowledgements: Special thanks to Kathryn Dzurilla for transcribing our patient interviews. We would also like to thank the nurses for their assistance.

\section{References}

1. Politi M, Han P, Col N. Communicating the uncertainty of harms and benefits of medical interventions. Medical Decision Making 2007 Sep-Oct;27(5):681-95.

2. Braddock C, Edwards K, Hasenberg N, Laidley T, Levinson W. Informed decision making in outpatient practice: time to get back to basics. JAMA. 1999;282(24):2313-20.

3. Han P, Klein W, Arora N. Varieties of uncertainty in health care: a conceptual taxonomy. Medical Decision Making 2011;31(6):828-838.

4. Han P. Conceptual, Methodological, and Ethical Problems in Communicating Uncertainty in Clinical Evidence. Med Care Res Rev 2013;70(10):14S-36S.

5. Ulkümen G, Fox, CR, Malle, BF. Two dimensions of subjective uncertainty: Clues from natural language. Journal of Experimental Psychology: General 2016;145:1280-1297.

6. Bansback N, Bell M, Spooner L, Pompeo A, Han PKJ, Harrison M. Communicating Uncertainty in Benefits and Harms: A Review of Patient Decision Support Interventions. The Patient: PatientCentered Outcomes Research. 2017;10(3):311-319.

7. Camerer $\mathrm{C}$, Weber M. Recent developments in modeling preferences: uncertainty and ambiguity. Journal of Risk and Uncertainty 1992;5:325-370.

8. Ellsberg D. Risk, ambiguity, and the savage axioms. Quarterly Journal of Economics 1961;75:643669.

9. Han P, Klein W, Lehman T, Killam B, Massett H, Freedman A. Communication of uncertainty regarding individualized cancer risk estimates: effects and influential factors. Medical Decision Making 2011;31:354-366.

10. Frosch D, Kaplan R, Felitti V. Evaluation of two methods to facilitate shared decision making for men considering the prostate-specific antigen test. J Gen Intern Med 2001;16:391-398.

11. Volk R, Cass A, Spann S. A randomized controlled trial of shared decision making for prostatespecific antigen screening. Arch Intern Med 1999;156:1333-1336.

12. Tannenbaum D, Fox C, Ülkümen G. Judgment extremity and accuracy under epistemic vs. aleatory uncertainty. Management Science 2017;63(2):497-518.

13. Mazur D, Hickam D. Patients' interpretations of probability terms. J Gen Intern Med 1991;6(3):237240. 
14. Bogardus S, Holmboe E, Jekel J. Perils, pitfalls, and possibilities in talking about medical risk. JAMA 1999;281:1037-1041.

15. Mazur D, Merz J. Patients' interpretations of verbal expressions of probability: implications for securing informed consent to medical interventions. Behav Sci Law 1994;12(4):417-26.

16. Peters E, Västfjäll D, Slovic P, Mertz C, Mazzocco K, Dickert S. Numeracy and decision making. Psychol Sci 2006;17:407-413.

17. Garcia-Retamero R, Galesic M. Communicating treatment risk reduction to people with low numeracy skills: A cross-cultural comparison. Am J Public Health 2009;99(12):2196-2202.

18. Reyna $\mathrm{V}$, Brainerd C. Numeracy, ratio bias, and denominator neglect in judgments of risk and probability. Learn Individ Differ 2008;18:89-107.

19. Peters E, SolHar P, Fraenkel L. Informing patients: the influence of numeracy, framing, and format of side effect information on risk perceptions. Medical Decision Making 2010;31(3):432-436.

20. Peters E, Hibbard J, Slovic P, Dieckmann N. Numeracy Skill And The Communication, Comprehension, And Use Of Risk-Benefit Information. Health Affairs 2007;26(3):741-748.

21. Cozmuta R, Wilhelms E, Cornell $D$, Nolte J, Reyna V, Fraenkel L. The influence of explanatory images on risk perceptions and treatment preference. Athritis Care Res (Hoboken) 2018;[Epub ahead of print].

22. Galesic M, Garcia-Retamero R, Gigerenzer G. Using icon arrays to communicate medical risks: overcoming low numeracy. Health Psychol 2009;28(2):210-216.

23. Galesic M, Garcia-Retamero R. Statistical numeracy for health: A cross-cultural comparison with probabilistic national samples. Arch Intern Med 2010;170(5):462-468.

24. Garcia-Retamero R, Galesic M. Who proficts from visual aids: Overcoming challenges in people's understanding of risks. Social Science \& Medicine 2010;70(7):1019-1025.

25. Gaissmaier W, Wegwarth O, Skopec D, Müller A, Broschinski S, Politi M. Numbers can be worth a thousand pictures: Individual differences in understanding graphical and numerical representations of health-related information. Health Psychology 2012;31(3):286-296.

26. Reyna V, Nelson W, Han P, NF D. How numeracy influences risk comprehension and medical decision making. Psychol Bull 2009;135(6):943-73.

27. Lipkus IM. Numeric, Verbal, and Visual Formats of Conveying Health Risks: Suggested Best Practices and Future Recommendations. Medical Decision Making 2007;27(5):696-713.

28. Ancker JS, Senathirajah Y, Kukafka R, Starren JB. Design Features of Graphs in Health Risk Communication: A Systematic Review. Journal of the American Medical Informatics Association 2006;13(6):608-618.

29. Eyler RF, Cordes S, Szymanski BR, Fraenkel L. Utilization of Continuous "Spinners" to Communicate Risk. Medical Decision Making 2017;37(6):725-729.

30. Keller C, Siegrist M. Effect of Risk Communication Formats on Risk Perception Depending on Numeracy. Medical Decision Making 2009;29(4):483-490. 
31. Reyna VF. A Theory of Medical Decision Making and Health: Fuzzy Trace Theory. Medical Decision Making 2008;28(6):850-865.

32. Fischhoff B, BruinedeBruin W. "Fifty-fifty" = 50\%? Journal of Behavioral Decision Making 1999;12:149-163.

33. Bruin WB, Fischbeck PS, Stiber N, Fischhoff B. What number is "fifty-fifty"?: Redistributing excessive $50 \%$ responses in elicited probabilities. Risk Analysis 2002;22(4):713-723.

34. BruinedeBruin W, Fischhoff B, Millstein S, Halpern-Felsher H. Verbal and numeric expressions of probability: "It's a 'fifty-fifty' chance.". Organizational Behavior and Human Processes 2000;81:115131.

35. Janssens A, deBoer J, Hintzen R, vanDoor P, Passcier J, vanderMeche F. "It might happen or not": patients' perceptions of prognostic risk in mulitiple sclerosis. Paper presented at the meeting of the Biannual Conference on Subjective Probability, Utility, and Decision Making. Amsterdam, the Netherlands. 2001. 